\title{
Knee Septic Arthritis after Arthroscopy: Incidence, Risk Factors, Functional Outcome, and Infection Eradication Rate
}

\author{
G. Balato ${ }^{1}$ S. L. Di Donato ${ }^{1} \quad$ T. Ascione ${ }^{2} \quad$ A. D’Addona ${ }^{1} \quad$ F. Smeraglia ${ }^{1} \quad$ G. Di Vico ${ }^{3}$ D. Rosa ${ }^{1}$ \\ ${ }^{1}$ Orthopaedic Unit, Department of Public Health, School of Medicine, \\ University of Naples Federico II, Naples, Italy \\ 2 Department of Infectious Diseases, D. Cotugno Hospital, \\ Naples, Italy \\ Address for correspondence G. Balato, MD, Department of Public \\ Health, School of Medicine, University of Naples Federico II, Via Sergio \\ Pansini 5, Building 12, 80131 Naples, Italy \\ (e-mail: giovannibalato@gmail.com).
}

${ }^{3}$ Orthopaedic Unit, S. Michele Clinic, Caserta, Italy

Joints 2017;5:107-113.

\begin{abstract}
Purpose Septic knee arthritis following arthroscopy is a rare but dreaded complication. Definition and management of knee deep infections are quite discussed in literature. In this review, literature regarding infections after knee arthroscopy is analyzed highlighting the incidence, causative bacteria, risk factors as well as clinical outcomes.

Methods We performed a review of the literature matching the following key words: "septic arthritis" OR “infection" AND "arthroscopy" AND "knee." Knee arthroscopic procedures, such as debridement, meniscectomy, meniscus repair, synovectomy, microfracture, and lateral release, were considered. Complex procedures, such as ligament reconstruction, fractures, or complex cartilage repair techniques, were not included.

Results Thirteen studies were included in this review. Incidence of infection ranged from 0.009 to $1.1 \%$ in patients undergoing simple arthroscopic procedures. Staphylococci are the most commonly isolated organisms from postarthroscopy infection. Use of intraoperative intra-articular steroids, smoking, obesity, male sex, diabetes, number of procedures performed during surgery, time of surgery, and tourniquet time

Keywords

- infection

- septic arthritis

- knee

- arthroscopy

- risk factors of more than 60 minutes have been certified as risk factors for knee infection.

Conclusion Postarthroscopy septic arthritis of the knee causes significant morbidity, usually requiring readmission to the hospital, at least one additional operation, and prolonged antibiotic therapy, both intravenous and oral. Prompt diagnosis and treatment are associated with a high success rate.

Level of Evidence Level IV, systematic review of I-IV studies.
\end{abstract}

\section{Introduction}

Arthroscopic knee surgery is one of the most common orthopaedic procedures performed worldwide, and would seem intrinsically "low risk" for infectious complications. ${ }^{1,2}$ Although the incidence of infection after arthroscopy is very low, ranging from 0.009 to $0.4 \%,{ }^{3-5}$ septic arthritis represents a potentially devastating postoperative complication, with possible sequelae including accelerated joint degeneration, early arthroplasty, fusion, and amputation with significant morbidity. Commonly involved organisms include Staphylococcus aureus and coagulase-negative staphylococci, while gram-negative bacteria are rarely found.

In this article, we reviewed the infectious complications of simple knee arthroscopy, and evaluated incidence rates, microbiological aspects, risk factors, treatment practices, 


\section{0 articles screened}

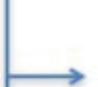

492 studies excluded after title/Abstract reviewed: review article (7), letters (2), studies not related to the research (474), no English language (4), no abstract available (5)

\section{Full-Text studies reviewed}

45 studies excluded: case report (6); studies not related to the research (28); studies not evaluating infection outcome (11)

\section{3 studies included in review}

Fig. 1 Flow chart of randomized controlled trials and cohort studies identified for the review.

and outcomes. Simple knee arthroscopy, including debridement, meniscectomy, meniscus repair, synovectomy, microfracture, and lateral release, were considered. We did not include complex procedures such as ligament reconstruction, fractures, or complex cartilage repair techniques.

\section{Methods}

To identify relevant papers dealing with knee infection after "simple" arthroscopic surgical procedures, we performed a research of literature on arthroscopy in English language using the MEDLINE database with the search strings "septic arthritis" OR "infection" AND "arthroscopy" AND "knee." Additional articles were identified by checking the references. Two authors (G.B. and S.L.D.D.) independently reviewed each abstract. Once a paper was identified as likely to be included, fulltext versions were obtained. Conflict about the inclusion of a paper was resolved by further evaluation, which was undertaken by the senior author (D.R.).

\section{Results}

A total of 550 published papers were extracted. Overall, 537 papers were excluded from the analysis for the following reasons: 7 were review articles, 4 were not in English, 6 were case reports, 502 studies were not related to the research, 11 studies did not evaluate the infectious outcome, 2 were letters to the editor, and 5 papers had a missing abstract. Thirteen papers were finally included (-Fig. $\mathbf{1}$ ).

\section{Incidence}

Reported rates of infection after "simple" arthroscopic knee surgery ranged from $0.009^{3}$ to $1.1 \%^{6}$ ( - Table 1). Unfortunately, there was no consensus on the definition of postsurgical septic arthritis in any of the selected papers. The definition of deep infection was specified only in three studies, wherein patients were considered infected only if there was a positive joint-fluid culture. ${ }^{4,5,7}$ Many of the studies reporting surgical site infection (SSI) were published before publication of the Centers for disease control and prevention (CDC) guidelines in 1992, which defined postoperative wound infections as superficial, incisional, deep incisional, and organ or space SSI. In the 1980s,

Table 1 Rate of postarthroscopic knee septic arthritis reported in selected studies

\begin{tabular}{|c|c|c|c|}
\hline Study/Reference & $\begin{array}{l}\text { Cases } \\
(N)\end{array}$ & $\begin{array}{l}\text { Infection } \\
\text { rate }(\%)\end{array}$ & Comments \\
\hline Clement et $\mathrm{al}^{14}$ & 975 & 0.19 & $\begin{array}{l}\text { Deep infection rate: } 0.22 \text { for all arthroscopic knee procedures, } 0.36 \text { for } \\
\text { high-complexity procedures }\end{array}$ \\
\hline Ashraf et al $^{13}$ & 3 & 0.3 & Deep infection after high- and low-complexity arthroscopic procedures \\
\hline Wyatt et al ${ }^{15}$ & 36 & 0.09 & Deep infection rate: 0.08 for antibiotic group, 0.14 for no-antibiotic group \\
\hline Hagino et $\mathrm{al}^{12}$ & 2 & 0.08 & \\
\hline Yeranosian et al ${ }^{11}$ & & 0.23 & The incidence of infection for diagnostic arthroscopy was 0.33 \\
\hline Balabaud et al ${ }^{10}$ & 2 & NA & \\
\hline Bert et $\mathrm{al}^{4}$ & 4 & 0.12 & $\begin{array}{l}\text { The infection rate was } 0.15 \% \text { in those patients who received antibiotics and } \\
0.16 \% \text { in those who did not receive antibiotics }\end{array}$ \\
\hline Johnson et al ${ }^{3}$ & 1 & 0.009 & Infection rate: $0.01 \%$ for diagnostic procedures, $0.04 \%$ for total procedures \\
\hline De Lee et $\mathrm{al}^{8}$ & 95 & 0.08 & \\
\hline Small et al ${ }^{9}$ & 11 & 0.36 & The overall infection rate was 0.28 including anterior cruciate procedure \\
\hline Sherman et $\mathrm{al}^{7}$ & NA & 0.1 & Infection defined as purulent joint fluid and positive result of joint-fluid culture \\
\hline Armstrong et $\mathrm{al}^{5}$ & 17 & 0.4 & $\begin{array}{l}\text { The overall infection rate was } 0.42 \text { including cruciate procedure and arthro- } \\
\text { scopy combined with an unrelated extra-articular surgical procedure }\end{array}$ \\
\hline Armstrong and Bolding 6 & 4 & 1.1 & \\
\hline
\end{tabular}

Abbreviation: NA, not applicable. 
there were four published articles about the complications subsequent to knee arthroscopy. Two national surveys were performed by the Complications Committee of the Arthroscopy Association of North America. These surveys were based on the responding physicians' recall of all complications including infections associated with arthroscopic procedures and the total number of arthroscopic procedures they had performed.

In 1985, DeLee $^{8}$ reported that $0.08 \%$ (95 cases) of recalled cases required drainage and antibiotic for intra-articular infection. Postsurgical infection represents the most frequent complication (10.2\% of all complications) after postoperative hemarthrosis, broken instruments, and thrombophlebitis.

Small ${ }^{9}$ reported a higher postsurgical knee infection rate of $0.28 \%$; when we considered deep infections and knee arthroscopy excluding anterior cruciate procedures, this rate increased to 0.36 ( 11 infections out of 3,034 procedures).

Sherman et $\mathrm{al}^{7}$ reviewed a large series of knee arthroscopies performed by a few surgeons in one orthopaedic group over a prolonged period. They defined infection as purulent fluid aspirated from the knee from which one organism was isolated, and reported an infection rate of $0.1 \%$.

Johnson et $\mathrm{al}^{3}$ reporting on the use of $2 \%$ glutaraldehyde for disinfecting arthroscopes between procedures, noted an overall infection rate of $0.04 \%$ ( 5 infections out of 12,505 procedures performed during 8 years): $0.01 \%$ for diagnostic arthroscopy and $0.009 \%$ for operative arthroscopy excluding open or reconstructive surgery. Septic arthritis developed in only one patient after diagnostic arthroscopy, which had been performed to determine the extent of loosening of a cemented knee prosthesis, and in four patients who underwent combined arthroscopy, arthrotomy, and capsular reconstruction.

Armstrong and Bolding ${ }^{6}$ reported an elevated rate of septic arthritis following arthroscopy at an institution, where 4 infections occurred out of 101 arthroscopic procedures performed during a 3-month period (infection rate, $4 \%)$. Three infections occurred after knee arthroscopy. During the subsequent 6 months, three additional postarthroscopic infections occurred among 251 arthroscopic procedures; so, the rate decreased to $1.2 \%$. Review of the combined data from the entire 9-month observation period from May 1990 through January 1991 showed an overall knee infection rate of $1.1 \%$. Previously, data on all postarthroscopic septic arthritis during a 4-year study were reported by Armstrong et $\mathrm{al}^{5}$ on more than 4,000 knee arthroscopies. Eighteen patients became infected (infection rate: 0.42), but the infection rate dropped to $0.4 \%$ when cruciate ligament reconstructions were excluded.

More recently, Bert et $\mathrm{al}^{4}$ retrospectively compared the incidence of knee-joint sepsis after routine arthroscopic surgery of the knee with and without preoperative intravenous antibiotics at a single outpatient surgery center over a 3-year period. The infection rate was $0.12 \%$ and in particular, $0.15 \%$ for those patients who received prophylactic antibiotics before the arthroscopic surgical procedure and $0.16 \%$ for those patients who did not receive antibiotics. In the same year, Balabaud et $\mathrm{al}^{10}$ described only four cases of infection after knee arthroscopy (one anterior cruciate replacement, one quadriceps tendon suture, and two "simple" arthroscopies).

Yeranosian et $\mathrm{al}^{11}$ determined the incidence of infection requiring reoperation after arthroscopic knee surgery during a 6-year period, comparing infection rates across different age groups, genders, geographic regions of the United States, and Current Procedural Terminology (CPT) codes through a retrospective review of a large insurance company database. The incidence of septic arthritis, excluding complex knee arthroscopy, ranged from 0.12 (arthroscopy with meniscectomy including any meniscal shaving) to 0.40 (arthroscopy with lysis of adhesions).

In the last 2 years, there have been four reported articles published on overall complications related to knee arthroscopy. Hagino et $\mathrm{al}^{12}$ examined the complications of arthroscopic knee surgeries conducted in a Sports Medicine and Knee Center. Postoperative complications consisted of two cases of septic arthritis ( $0.008 \%$ of cases) and one case of superficial infection controlled by arthroscopic washout and debridement.

Ashraf et $\mathrm{al}^{13}$ analyzed the incidence of acute and subacute complications of knee arthroscopy in patients aged 17 years or younger and identified the associated risk factors. Major complications were defined as events that were either life or limb threatening, required additional surgery, or had potentially detrimental effects on the patient's long-term outcome. The most common major complication was a wound complication that required surgical reassessment followed by arthrofibrosis requiring manipulation under anesthesia, unplanned revision surgery, and septic arthritis requiring irrigation and debridement $(0.3 \%)$. This percentage was related to high- and low-complexity arthroscopic procedures. In contrast, Clement et $\mathrm{al}^{14}$ showed a deep infection rate of 0.19 and $0.36 \%$ in low- and high-complexity procedures, respectively.

Wyatt et $\mathrm{al}^{15}$ determined the effect of administering a preoperative antibiotic to patients undergoing knee arthroscopy on the incidence of postoperative infection. There were 36 deep infections (0.09\%): 25 in the antibiotic group (0.08\%) and 11 in the no-antibiotic group (0.14\%).

\section{Microbiology and Risk Factors}

The causative bacteria were reported only in four papers (-Table 2). In the majority of reports, the pathogen most commonly identified from surgical site infections that occur after arthroscopy was Staphylococcus, both coagulase positive and negative. Other infections have been reported to include Enterobacter cloacae (6 patients), Streptococcus spp. (6 patients), and Serratia marcescens ( 3 patients); 11 patients had culture-negative infections.

Risk factors for infection have been addressed in multiple studies although they usually have been identified through surveillance analysis, with or without comparison with controls. Several risk factors have been suggested by this uncontrolled data including preoperative risk factors, factors related to the host, and surgical risk factors (-Table 3 ).

The use of intraoperative intra-articular steroids has been discussed frequently as a risk factor for infection. ${ }^{5,16}$ 
Table 2 Microbiologic findings from articles reviewed

\begin{tabular}{|c|c|c|c|}
\hline & Microorganism isolated & Percentage (\%) & Comments \\
\hline \multirow[t]{7}{*}{ Wyatt et al ${ }^{15}$} & Coagulase-negative Staphylococcus & 36 & \\
\hline & Staphylococcus aureus & 27 & \\
\hline & MRSA & 11 & \\
\hline & Enterobacteria & 6 & \\
\hline & Streptococcus spp. & 6 & \\
\hline & Serratia marcescens & 3 & \\
\hline & Culture negative & 11 & \\
\hline Balabaud et al $^{10}$ & NA & & $\begin{array}{l}\text { The most frequently isolated mi- } \\
\text { croorganism in all cases is MSSA } \\
\text { but is not specified in postar- } \\
\text { throscopic septic arthritis } \\
\text { patients }\end{array}$ \\
\hline Bert et $\mathrm{al}^{4}$ & Staphylococcus aureus & 100 & \\
\hline Johnson et $\mathrm{al}^{3}$ & Staphylococcus aureus & 100 & \\
\hline Small et $\mathrm{al}^{9}$ & NA & & $\begin{array}{l}\text { Staphylococcus infection is the } \\
\text { most frequent }\end{array}$ \\
\hline Jonhson et $\mathrm{al}^{3}$ & Staphylococcus aureus & 100 & \\
\hline \multirow[t]{3}{*}{ Armstrong et $\mathrm{al}^{5}$} & Staphylococcus aureus & 53 & \\
\hline & Coagulase-negative Staphylococcus & 41 & \\
\hline & Enterobacter cloacae & 6 & \\
\hline Armstrong and Bolding 6 & NA & & $\begin{array}{l}\text { Staphylococcus infection is the } \\
\text { most frequent }\end{array}$ \\
\hline
\end{tabular}

Abbreviations: MRSA, methicillin-resistant Staphylococcus aureus; MSSA, methicillin-sensitive Staphylococcus aureus; NA, not applicable.

Armstrong et $\mathrm{al}^{5}$ showed that use of intra-articular corticosteroids declined after the first 2 years of the study, and the risk of infection with its use was highly significant. These results were also confirmed later by Armstrong and Bolding in $1994 .^{6}$ The CDC recommends avoiding the use of intraoperative intra-articular steroids. ${ }^{17}$

The efficacy of prophylactic antibiotics in knee arthroscopy has been discussed in two papers. Wyatt et $\mathrm{al}^{15}$ in their large sample of patients who underwent simple knee arthroscopy, reported that there was no association between preoperative antibiotic use and postoperative deep or superficial infection rates. Similar results in terms of incidence of knee joint sepsis after routine arthroscopic surgery of the knee with and without preoperative intravenous antibiotics were reported by Bert et $\mathrm{al}^{4}{ }^{4}$ who performed a retrospective study on the incidence of infection after routine arthroscopic meniscectomy with and without prophylactic antibiotics. Over a 3-year period, 2,780 arthroscopic cases were collected. Of the 933 patients who received an antibiotic, 1 $(0.11 \%)$ had a deep infection. Of the 1,847 patients who did not receive an antibiotic, $3(0.16 \%)$ had a deep infection. Although these results were not statistically significant, this study had an insufficient sample size to rule out a clinically significant difference. The results of this study confirmed that there is no value in administering antibiotics before routine arthroscopic meniscectomy to prevent joint sepsis.

Table 3 Risk factors

\begin{tabular}{|c|c|}
\hline Sherman et $\mathrm{al}^{7}$ & Patient age $>50 \mathrm{y}$, tourniquet time $>60 \mathrm{~min}$ \\
\hline Armstrong et $\mathrm{al}^{5}$ & Administration of intra-articular corticosteroids, length of surgery \\
\hline Armstrong and Bolding ${ }^{6}$ & Administration of intra-articular corticosteroids \\
\hline Clement et al ${ }^{14}$ & $\begin{array}{l}\text { Age, } 15-49 \text { y; male; diabetic; morbidly obese; tobacco use; high-complexity procedure; in } \\
\text { low-complex procedure: patient age }<50 \text { y and male sex }\end{array}$ \\
\hline Wyatt et al ${ }^{15}$ & $\begin{array}{l}\text { White patients (univariate regression analysis); patient age }>40 \text { y (univariate and } \\
\text { multivariate regression analyses) }\end{array}$ \\
\hline Yeranosian et al ${ }^{11}$ & Male sex, patient age $<60 \mathrm{y}$ \\
\hline
\end{tabular}


The study by Clement et al $^{14}$ attempted to identify and quantify patient- and procedure-related risk factors for postarthroscopic knee infections using a large dataset. They found that there were higher rates of deep, superficial, and total infections among tobacco users, patients undergoing high-complexity procedures, morbidly obese patients, men, diabetic patients, and patients younger than 50 years. Tobacco use was the strongest predictor of deep infections, followed by procedure complexity, morbid obesity, diabetes, age younger than 50 years, and male sex. Again, a higher rate of deep infections was observed among younger men (age $<50$ years) undergoing low-complexity procedures.

Similar results were previously reported by Yeranosian et al, ${ }^{11}$ who found that male sex and younger age were associated with infections but did not stratify by deep, superficial, and total infection rate, nor did they consider additional risk factors known to predict surgical-site infections in other areas of orthopaedics, including tobacco use, body mass index (BMI), and diabetes. The age at surgery, like the risk factor, was confirmed by Bert et $\mathrm{al}^{4}$ and Sherman et $\mathrm{al}^{7}$ who found that age more than 40 years was associated with an increased risk of infection. The other variables studied (race, gender, BMI, American Society of Anesthesiologists [ASA] status, diabetes, operative time) were not associated with risk of postoperative infection.

Other risk factors such as length of surgery, number of procedures performed during surgery, and type of procedures were studied. Time of surgery and tourniquet time of more than 60 minutes were statistically significant factors for increased risk of postoperative infection. ${ }^{5,7}$ Armstrong et $\mathrm{al}^{5}$ reported that the length of surgery was significantly longer for infected patients. In contrast, the number and type of surgical procedures have not been shown to have a statistically significant correlation with the occurrence of a postoperative infection. In addition, Sherman et $\mathrm{al}^{7}$ showed that the tourniquet time was a dominant predictor of com- plications. The overall complication rate for the procedures in which a tourniquet was used was $8.9 \%$, with $5.4 \%$ major and $3.7 \%$ minor complications, but Sherman's group did not separately differentiate infections from overall major complications.

\section{Treatment and Outcome}

The treatment modalities were clearly stated in only six papers (-Table 4). Most patients were successfully treated with a combination of antibiotic therapy and surgical intervention. All patients with more significant infections received intravenous antibiotics for prolonged courses, ranging from 15 days ${ }^{10}$ to 4 weeks. ${ }^{4}$ For some patients, intravenous antibiotic therapy was followed by another 2 to 4 weeks of oral antibiotic therapy. ${ }^{5,10}$ Oral antibiotics were discontinued when all clinical signs of septic arthritis had disappeared and when the $\mathrm{C}$-reactive protein had returned to normal values. ${ }^{10}$ Most patients required irrigation and lavage of the joint, either arthroscopically or by means of open arthrotomy associated with antibiotics, 4,5,10,12 although some were managed with needle aspirations followed by 2 weeks of intravenous antibiotics. ${ }^{5,8}$ In one case with a knee infection, open drainage of the joint and antibiotic therapy were required. ${ }^{3}$ Armstrong et $\mathrm{al}^{5}$ reported four cases treated with needle aspiration and intravenous antibiotic treatment showing good clinical results.

Not all reviewed articles included outcome information (-Table 4). The treatment was successful in all cases analyzed with an infection eradication rate of $100 \%$. However, two papers reported decreased function of the involved joint. De Lee et $\mathrm{al}^{8}{ }^{8}$ in their national survey, reported that of those patients who developed a postoperative infection, 18 patients had a poorer outcome with decreased knee flexion (less than 90 degrees). Armstrong et $\mathrm{al}^{5}$ reported that none of their patients had a secondary infection or immediate relapse with long-term excellent results in $73 \%$ of patients.

Table 4 Treatment modalities and outcome

\begin{tabular}{|c|c|c|c|}
\hline Author & Treatment & $\begin{array}{l}\text { Duration of } \\
\text { antibiotic } \\
\text { treatment }\end{array}$ & Outcome \\
\hline Hagino et al $^{12}$ & Arthroscopic debridement + antibiotic therapy & NA & $100 \%$ cured \\
\hline \multicolumn{4}{|l|}{${\text { Balabaud et } \text { al }^{10}}^{10}$} \\
\hline Bert et $\mathrm{al}^{4}$ & Single arthroscopic debridement + antibiotic therapy (IV) & $4-6$ wk & $100 \%$ cured \\
\hline Johnson et $\mathrm{al}^{3}$ & Open drainage + antibiotic therapy & NA & $100 \%$ cured \\
\hline De Lee et $\mathrm{al}^{8}$ & Drainage + antibiotic therapy & NA & $\begin{array}{l}\text { 18.9\% had poor } \\
\text { outcome with de- } \\
\text { creased knee flex- } \\
\text { ion (less than } 90 \\
\text { degrees) }\end{array}$ \\
\hline \multirow[t]{2}{*}{ Armstrong et $\mathrm{al}^{5}$} & Debridement + antibiotic therapy (IV + per OS) & NA & $\begin{array}{l}100 \% \text { cured (73\% } \\
\text { excellent results) }\end{array}$ \\
\hline & Needle aspiration + antibiotic therapy (per OS) in two patients & $2 \mathrm{wk}$ & $\begin{array}{l}100 \% \text { cured (good } \\
\text { results) }\end{array}$ \\
\hline
\end{tabular}

Abbreviations: NA, not applicable; OS, by mouth. 
Although all patients were cured, some reported a decreased knee function. They also reported good clinical results in four cases treated with only needle aspiration and 2 weeks of intravenous antibiotic therapy.

Because these studies were not controlled trials designed to assess validated outcome measures, it is difficult to draw conclusion; however, clearly, infectious complications can be associated with some disability after these usually welltolerated procedures. ${ }^{18,19}$

\section{Discussion}

Septic arthritis after knee arthroscopy is a very uncommon complication. Data from literature indicate an incidence rate ranging from 0.009 to $1.1 \%$ in patients undergoing simple arthroscopic procedures. Unfortunately, there was no consensus on the definition of postsurgical septic arthritis in all the reviewed papers. The definition of deep infection was only specified in three studies in which patients were considered infected only in case of a positive joint-fluid culture. ${ }^{4,5,7}$ For this reason, it was difficult to arrive at any crucial conclusion regarding the incidence, risk factors, and treatment. In this review, we considered only those cases that underwent simple knee arthroscopy including diagnostic arthroscopy, debridement, meniscectomy, meniscus repair, synovectomy, microfracture, and lateral release.

Infections in immunocompromised people are generally difficult to diagnose due to aspecific symptoms, but patients undergoing knee arthroplasty are generally in good health and infections sustained by opportunistic agents including parasites are never reported and hospital-acquired bacteria are reported with the highest frequency. Moreover, fever itself cannot be considered an accurate symptom related to infection. ${ }^{20-23}$

Staphylococci remain the most commonly isolated microorganisms from postarthroscopy SSI. ${ }^{3-6,9,10,15}$ In 11 cases with deep infections, the causative microorganism was unknown. Risk factors for infection have been addressed in multiple studies although they usually have been identified through surveillance analysis, with or without comparison with controls. The use of intraoperative intra-articular steroids has been certified as a risk factor for infection in two of the reported papers. ${ }^{5,16}$ Tobacco users, patients undergoing high-complexity procedures, morbidly obese patients, men, diabetic patients, and patients younger than 50 years are predisposed to a higher incidence of superficial and deep infections. Other risk factors, such as length of surgery, number of procedures performed during surgery, and type of procedures, were studied. Time of surgery and tourniquet time of more than 60 minutes were statistically significant factors for increased risk of postoperative infection.

Factors encountered are quite different than those reported to be associated with the risk of infection in patients undergoing joint arthroplasty. ${ }^{24,25}$

In conclusion, although uncommon, postarthroscopy SSI do cause significant morbidity in patients, usually requiring readmission to the hospital, at least one additional operation, and prolonged antibiotic therapy, both intravenous and oral.
The treatment has been successful in all cases analyzed, with an infection eradication rate of $100 \%$. However, two papers reported decreased function of the involved joint. ${ }^{5,8}$

\section{References}

1 Kim S, Bosque J, Meehan JP, Jamali A, Marder R. Increase in outpatient knee arthroscopy in the United States: a comparison of National Surveys of Ambulatory Surgery, 1996 and 2006. J Bone Joint Surg Am 2011;93(11):994-1000

2 Lubowitz JH, Ayala M, Appleby D. Return to activity after knee arthroscopy. Arthroscopy 2008;24(01):58-61.e4

3 Johnson LL, Shneider DA, Austin MD, Goodman FG, Bullock JM, DeBruin JA. Two per cent glutaraldehyde: a disinfectant in arthroscopy and arthroscopic surgery. J Bone Joint Surg Am 1982;64 (02):237-239

4 Bert JM, Giannini D, Nace L. Antibiotic prophylaxis for arthroscopy of the knee: is it necessary? Arthroscopy 2007;23(01):4-6

5 Armstrong RW, Bolding F, Joseph R. Septic arthritis following arthroscopy: clinical syndromes and analysis of risk factors. Arthroscopy 1992;8(02):213-223

6 Armstrong RW, Bolding F. Septic arthritis after arthroscopy: the contributing roles of intraarticular steroids and environmental factors. Am J Infect Control 1994;22(01):16-18

7 Sherman OH, Fox JM, Snyder SJ, et al. Arthroscopy-“no-problem surgery". An analysis of complications in two thousand six hundred and forty cases. J Bone Joint Surg Am 1986;68(02): 256-265

8 DeLee JC, Elliot B, Fiddan N, et al; Committee on Complications of Arthroscopy Association of North America. Complications of arthroscopy and arthroscopic surgery: results of a national survey. Arthroscopy 1985;1(04):214-220

9 Small NC, Cohen S, Ehlich G, et al; Committee on Complications of the Arthroscopy Association of North America. Complications in arthroscopy: the knee and other joints. Arthroscopy 1986;2(04): 253-258

10 Balabaud L, Gaudias J, Boeri C, Jenny JY, Kehr P. Results of treatment of septic knee arthritis: a retrospective series of 40 cases. Knee Surg Sports Traumatol Arthrosc 2007;15(04): 387-392

11 Yeranosian MG, Petrigliano FA, Terrell RD, Wang JC, McAllister DR. Incidence of postoperative infections requiring reoperation after arthroscopic knee surgery. Arthroscopy 2013;29(08):1355-1361

12 Hagino T, Ochiai S, Watanabe Y, et al. Complications after arthroscopic knee surgery. Arch Orthop Trauma Surg 2014;134(11): 1561-1564

13 Ashraf A, Luo TD, Christophersen C, Hunter LR, Dahm DL, McIntosh AL. Acute and subacute complications of pediatric and adolescent knee arthroscopy. Arthroscopy 2014;30(06):710-714

14 Clement RC, Haddix KP, Creighton RA, Spang JT, Tennant JN, Kamath GV. Risk factors for infection after knee arthroscopy: analysis of 595,083 cases from 3 United States databases. Arthroscopy 2016;32(12):2556-2561

15 Wyatt RW, Maletis GB, Lyon LL, Schwalbe J, Avins AL. Efficacy of prophylactic antibiotics in simple knee arthroscopy. Arthroscopy 2017;33(01):157-162

16 Montgomery SC, Campbell J. Septic arthritis following arthroscopy and intra-articular steroids. J Bone Joint Surg Br 1989;71 (03):540-540

17 Mangram AJ, Horan TC, Pearson ML, Silver LC, Jarvis WR; Hospital Infection Control Practices Advisory Committee. Guideline for prevention of surgical site infection, 1999. Infect Control Hosp Epidemiol 1999;20(04):250-278; quiz 279-280

18 Babcock HM, Matava MJ, Fraser V. Postarthroscopy surgical site infections: review of the literature. Clin Infect Dis 2002;34(01): 65-71 
19 Bert JM, Bert TM. Management of infections after arthroscopy. Sports Med Arthrosc Rev 2013;21(02):75-79

20 Pagliano P, Costantini S, Gradoni L, et al. Distinguishing visceral leishmaniasis from intolerance to pegylated interferon-alpha in a thalassemic splenectomized patient treated for chronic hepatitis C. Am J Trop Med Hyg 2008;79(01):9-11

21 Pagliano P, Carannante N, Gramiccia M, et al. Visceral leishmaniasis causes fever and decompensation in patients with cirrhosis. Gut 2007;56(06):893-894

22 Ascione T, Balato G, Pagliano P. Perspective: How to deal with fever $\left(38^{\circ} \mathrm{C}\right)$ after arthroplasty: the infectivologist's point of view. In: Baldini A, Caldora P, eds. Perioperative Medical Management for Total Joint Arthroplasty: How to Control Hemostasis, Pain and
Infection. Switzerland: Springer International Publishing; 2015: 297-301

23 Ascione T, Balato G, Boccia G, De Caro F. Predictive value of fever following arthroplasty in diagnosing an early infection. Infez Med 2017;25(01):3-7

24 Ascione T, Pagliano P, Balato G, Mariconda M, Rotondo R, Esposito S. Oral therapy, microbiological findings, and comorbidity influence the outcome of prosthetic joint infections undergoing 2-stage exchange. J Arthroplasty 2017. Doi: 10.1016/j.arth.2017.02.057

25 Ascione T, Pagliano P, Mariconda M, et al. Factors related to outcome of early and delayed prosthetic joint infections. J Infect 2015;70(01):30-36 